\title{
Short simplex paths in lattice polytopes
}

\author{
Alberto Del Pia * Carla Michini ${ }^{\dagger}$
}

April 9, 2020

\begin{abstract}
The goal of this paper is to design a simplex algorithm for linear programs on lattice polytopes that traces 'short' simplex paths from any given vertex to an optimal one. We consider a lattice polytope $P$ contained in $[0, k]^{n}$ and defined via $m$ linear inequalities. Our first contribution is a simplex algorithm that reaches an optimal vertex by tracing a path along the edges of $P$ of length in $O\left(n^{4} k \log (n k)\right)$. The length of this path is independent from $m$ and it is the best possible up to a polynomial function. In fact, it is only polynomially far from the worst-case diameter, which roughly grows as a linear function in $n$ and $k$.

Motivated by the fact that most known lattice polytopes are defined via $0, \pm 1$ constraint matrices, our second contribution is an iterative algorithm which exploits the largest absolute value $\alpha$ of the entries in the constraint matrix. We show that the length of the simplex path generated by the iterative algorithm is in $O\left(n^{2} k \log (n k \alpha)\right)$. In particular, if $\alpha$ is bounded by a polynomial in $n, k$, then the length of the simplex path is in $O\left(n^{2} k \log (n k)\right)$.

For both algorithms, the number of arithmetic operations needed to compute the next vertex in the path is polynomial in $n, m$ and $\log k$. If $k$ is polynomially bounded by $n$ and $m$, the algorithm runs in strongly polynomial time.
\end{abstract}

Key words: lattice polytopes; simplex algorithm; diameter; strongly polynomial time

\section{Introduction}

Linear programming (LP) is one of the most fundamental types of optimization models. In an LP problem, we are given a polyhedron $P \subseteq \mathbb{R}^{n}$ and a cost vector $c \in \mathbb{Z}^{n}$, and we wish to solve the optimization problem

$$
\max \left\{c^{\top} x \mid x \in P\right\} .
$$

The polyhedron $P$ is explicitly given via a system of linear inequalities, i.e., $P=\{x \in$ $\left.\mathbb{R}^{n} \mid A x \leq b\right\}$, where $A \in \mathbb{Z}^{m \times n}, b \in \mathbb{Z}^{m}$. If $P$ is nonempty and bounded, problem (11) admits an optimal solution that is a vertex of $P$.

${ }^{*}$ Department of Industrial and Systems Engineering \& Wisconsin Institute for Discovery, University of Wisconsin-Madison, Madison, WI, USA. E-mail: delpia@wisc.edu.

${ }^{\dagger}$ Department of Industrial and Systems Engineering, University of Wisconsin-Madison, Madison, WI, USA. E-mail: michini@wisc.edu. 
In this paper, we consider a special class of linear programs (11) where the feasible region is a lattice polytope, i.e., a polytope whose vertices have integer coordinates. These polytopes are particularly relevant in discrete optimization and integer programming, as they correspond to the convex hull of the feasible solutions to such optimization problems. In particular, a $[0, k]$-polytope in $\mathbb{R}^{n}$ is defined as a lattice polytope contained in the box $[0, k]^{n}$.

One of the main algorithms for LP is the simplex method. The simplex method moves from the current vertex to an adjacent one along an edge of the polyhedron $P$, until an optimal vertex is reached or unboundedness is detected, and the selection of the next vertex depends on a pivoting rule. The sequence of vertices generated by the simplex method is called the simplex path. The main objective of this paper is to design a simplex algorithm for $[0, k]$-polytopes that constructs 'short' simplex paths from any starting vertex $x^{0}$.

But how short can a simplex path be? A natural lower bound on the length of a simplex path from $x^{0}$ to $x^{*}$ is given by the distance between these two vertices, which is defined as the minimum length of a path connecting $x^{0}$ and $x^{*}$ along the edges of the polyhedron $P$. The diameter of $P$ is the largest distance between any two vertices of $P$, and therefore it provides a lower bound on the length of a worst-case simplex path on $P$. It is known that the diameter of $[0,1]$-polytopes in $\mathbb{R}^{n}$ is at most $n[22$ and this bound is attained by the hypercube $[0,1]^{n}$. This upper bound was later generalized to $n k$ for general $[0, k]$-polytopes in $\mathbb{R}^{n}[18]$, and refined to $n\left\lfloor\left(k-\frac{1}{2}\right)\right\rfloor$ for $k \geq 2[8]$ and to $n k-\left\lceil\frac{2}{3} n\right]-(k-3)$ for $k \geq 3[10]$. For $k=2$ the bound given in [8] is tight. In general, for fixed $k$, the diameter of lattice polytopes can grow linearly with $n$, since there are lattice polytopes, called primitive zonotopes, that can have diameter in $\Omega(n)$ [9]. Viceversa, when $n$ is fixed, the diameter of a $[0, k]$-polytope in $\mathbb{R}^{n}$ can grow almost linearly with $k$. In fact, it is known that for $n=2$ there are $[0, k]$-polytopes with diameter in $\Omega\left(k^{2 / 3}\right)$ [4, 27, 1]. Moreover, for any fixed $n$, there are primitive zonotopes with diameter in $\Omega\left(k^{\frac{n}{n+1}}\right)$ for $k$ that goes to infinity [1].

Can we design a simplex algorithm whose simplex path length is only polynomially far from optimal, meaning that it is upper bounded by a polynomial function of the worst-case diameter? In this paper, we answer this question in the affermative. Our first contribution is a preprocessing \& scaling algorithm that generates a simplex path of length in $O\left(n^{4} k \log (n k)\right)$ for $[0, k]$-polytopes in $\mathbb{R}^{n}$. The length of the simplex path is indeed polynomially far from optimal, as it is polynomially bounded in $n$ and $k$. We remark that the upper bound is independent on $m$. This is especially interesting because, even for $[0,1]$-polytopes, $m$ can grow exponentially in $n$ (see, e.g., [24]).

Our next objective is that of decreasing the gap between the length $O\left(n^{4} k \log (n k)\right)$ provided by the preprocessing \& scaling algorithm and the worst-case diameter, for wide classes of $[0, k]$-polytopes. We focus our attention on $[0, k]$-polytopes with bounded parameter $\alpha$, defined as the largest absolute value of the entries in the constraint matrix $A$. This assumption is based on the fact that the overwhelming majority of $[0, k]$-polytopes arising in combinatorial optimization for which an external description is known, satisfies $\alpha=1$ [24]. Our second contribution is another simplex algorithm, named the iterative algorithm, which generates a simplex path of length in $O\left(n^{2} k \log (n k \alpha)\right)$. Thus, by exploiting the parameter $\alpha$, we are able to significantly improve the dependence on $n$. Moreover, 
the dependence on $\alpha$ is only logarithmic, thus if $\alpha$ is bounded by a polynomial in $n, k$, then the length of our simplex path is in $O\left(n^{2} k \log (n k)\right)$. For $[0,1]$-polytopes this bound reduces to $O\left(n^{2} \log n\right)$.

In both our simplex algorithms, our pivoting rule is such that the number of operations needed to construct the next vertex in the simplex path is bounded by a polynomial in $n$, $m$, and $\log k$. If $k$ is bounded by a polynomial in $n, m$, both our simplex algorithms are strongly polynomial. This assumption is justified by the existence of $[0, k]$-polytopes that, for fixed $n$, have a diameter that grows almost linearly in $k$ [11]. Consequently, in order to obtain a simplex algorithm that is strongly polynomial also for these polytopes, we need to assume that $k$ is bounded by a polynomial in $n$ and $m$. We remark that in this paper we use the standard notions regarding computational complexity in Discrete Optimization, and we refer the reader to Section 2.4 in the book [23] for a thorough introduction.

\section{Overview of the algorithms and related work}

Our goal is to study the length of the simplex path in the setting where the feasible region of problem (11) is a $[0, k]$-polytope. As discussed above, our main contribution is the design of a simplex algorithm that visits a number of vertices polynomial in $n$ and $k$, from any starting vertex $x^{0}$. To the best of our knowledge, this question has not been previously addressed.

Since $P$ is a lattice polytope in $[0, k]^{n}$, it is not hard to see that a naive simplex algorithm, that we call the basic algorithm, always reaches an optimal vertex of $P$ by constructing a simplex path of length at most $n k\|c\|_{\infty}$. Therefore, in order to reach our goal, we need to make the simplex path length independent from $\|c\|_{\infty}$.

To improve the dependence on $\|c\|_{\infty}$ we design a scaling algorithm that recursively calls the basic algorithm with finer and finer integral approximations of $c$. By using this bit scaling technique 2], we are able to construct a simplex path to an optimal vertex of $P$ of length in $O\left(n k \log \|c\|_{\infty}\right)$.

The dependence of the simplex path length on $\|c\|_{\infty}$ is now logarithmic. Next, to completely remove the dependence on $\|c\|_{\infty}$, we first apply a preprocessing algorithm due to Frank and Tardos [14, that replaces $c$ with a cost vector $\breve{c}$ such that $\log \|\breve{c}\|_{\infty}$ is polynomially bounded in $n$ and $\log k$. By using this preprocessing step in conjunction with our scaling algorithm, we design a preprocessing \& scaling algorithm that constructs a simplex path whose length is polynomially bounded in $n$ and $k$.

Theorem 1. The preprocessing \& scaling algorithm generates a simplex path of length in $O\left(n^{4} k \log (n k)\right)$.

Our next task is to improve the polynomial dependence on $n$ by exploiting the knowledge of $\alpha$, the largest absolute value of an entry of $A$. In particular, we design an iterative algorithm which, at each iteration, identifies one constraint of $A x \leq b$ that is active at each optimal solution of (11). Such constraint is then set to equality, effectively restricting the feasible region of (11) to a lower dimensional face of $P$. At each iteration, we compute a suitable approximation $\tilde{c}$ of $c$ and we maximize $\tilde{c}^{\top} x$ over the current face of $P$. In order to solve this LP problem, we apply the scaling algorithm and we trace a path along the 
edges of $P$. We also compute an optimal solution to the dual, which is used to identify a new constraint of $A x \leq b$ that is active at each optimal solution of (11). The final simplex path is then obtained by merging together the different paths constructed by the scaling algorithm at each iteration.

Theorem 2. The iterative algorithm generates a simplex path of length in $O\left(n^{2} k \log (n k \alpha)\right)$.

Our iterative algorithm is inspired by Tardos' strongly polynomial algorithm for combinatorial problems [26. The three key differences with respect to Tardos' algorithm are: (1) Tardos' algorithm solves LP problems in standard form, while we consider a polytope $P$ in general form, i.e., $P=\left\{x \in \mathbb{R}^{n} \mid A x \leq b\right\}$; (2) Tardos' algorithm is parametrized with respect to the largest absolute value of a subdeterminant of the matrix A, while our algorithm relies on parameters $k$ and $\alpha$; and (3) Tardos' algorithm is not a simplex algorithm, while our algorithm traces a simplex path along the edges of $P$. Mizuno [20, 21] proposed a variant of Tardos' algorithm that traces a dual simplex path, under the assumption that $P$ is simple and that the constraint matrix is totally unimodular. We remark that the basic solutions generated by Mizuno's algorithm might not be primal feasible.

Next, we compare the length of the simplex path constructed by the iterative algorithm to the upper bounds that are known for some other classic pivoting rules. A result by Kitahara, Matsui and Mizuno [17, 16] implies that, if $Q=\left\{x \in \mathbb{R}_{+}^{n} \mid D x=d\right\}$ is a $[0, k]$-polytope in standard form with $D \in \mathbb{Z}^{p \times n}$ and $d \in \mathbb{Z}^{p}$, the length of the simplex path generated with Dantzig's or the best improvement pivoting rule is at most $(n-p)$. $\min \{p, n-p\} \cdot k \cdot \log (k \min \{p, n-p\})$. This has been recently extended to the steepest edge pivoting rule by Blanchard, De Loera and Louveaux [6].

Recall that in this paper we consider a lattice polytope $P \subseteq[0, k]^{n}$ in general form, i.e., $P=\left\{x \in \mathbb{R}^{n} \mid A x \leq b\right\}$. By applying an affine transformation, we can map $P$ to the standard form polytope $\bar{P}=\left\{(x, s) \in \mathbb{R}_{+}^{n+m} \mid A x+I_{m} s=b\right\}$, where $I_{m}$ denotes the identity matrix of order $m$. Note that $\bar{P}$ is a lattice polytope and it has the same adjacency structure of $P$. However, the affine transformation does not preserve the property of being a $[0, k]$-polytope. In fact, having introduced the slack variables $s$, we obtain that $\bar{P}$ is a lattice polytope in $[0, K]^{n+m}$, where $K=\max \{k, S\}$ and $S=\max \left\{\|b-A x\|_{\infty} \mid x \in P\right\}$. In this setting, the result of Kitahara, Matsui and Mizuno implies that we can construct a simplex path whose length is at most $n^{2} K \log (n K)$, but $K$ critically depends on $S$, which in turns depends on $A, b$. In fact, it is known that, even for $k=1$, the value $S$ can be as large as $\frac{(n-1)^{\frac{n-1}{2}}}{2^{2 n+o(n)}}$ (see [3, 28]), which is not polynomially bounded in $n, k$. In turn, this implies that the length of the simplex path obtained in this way for $[0, k]$-polytopes is not polynomially bounded in $n, k$.

In particular, if each inequality of $A x \leq b$ is active at some vertex of $P$, we have that $S$ and $K$ are in $O(n k \alpha)$, thus in this case the upper bound implied by the result of Kitahara, Matsui and Mizuno is in $O\left(n^{3} k \alpha \log (n k \alpha)\right)$. Note that the dependence on $\alpha$ is superlinear, while in our iterative algorithm it is only logarithmic. Even if $\alpha=1$, the upper bound is in $O\left(n^{3} k \log (n k)\right)$. In contrast, the upper bound given by the iterative algorithm is in $O\left(n^{2} k \log (n k)\right)$.

To show that the bound of $O(n k \alpha)$ on $S$ and $K$ just discussed can be tight, we now provide an example of a $[0,1]$-polytope with $\alpha=1$ and $S \in \Omega(n)$. Consider the stable set 
polytope of a $t$-perfect graph $G=(V, E)$, that is defined by the vectors $x \in \mathbb{R}_{+}^{V}$ satisfying:

$$
\begin{array}{rlrl}
x_{i}+x_{j} & \leq 1 & & i j \in E \\
\sum_{i \in V(C)} x_{i} \leq\left\lfloor\frac{\mid V(C)\rfloor}{2}\right\rfloor & & C \text { odd cycle in } G,
\end{array}
$$

where $V(C)$ denotes the nodes in the odd cycle $C$ [24]. Note that $x=0$ is the characteristic vector of the empty stable set, thus it is a vertex of the stable set polytope. If $G$ is an odd cycle on $|V|=n$ nodes, then $G$ is $t$-perfect, and the inequality (2) corresponding to the cycle containing all nodes of $G$ is facet-defining. Furthermore, the slack in such constraint can be as large as $\left\lfloor\frac{n}{2}\right\rfloor$, therefore $S \in \Omega(n)$. Consequently, the upper bound implied by [17, 16] is in $\Omega\left(n^{3} \log n\right)$, while the upper bound given by our iterative algorithm is in $O\left(n^{2} \log n\right)$.

\section{The preprocessing \& scaling algorithm}

In the remainder of the paper, we study problem (10), where $c$ is a given cost vector in $\mathbb{Z}^{n}$, and $P$ is a $[0, k]$-polytope given via a system of linear inequalities, i.e., $P=\left\{x \in \mathbb{R}^{n} \mid\right.$ $A x \leq b\}$, where $A \in \mathbb{Z}^{m \times n}, b \in \mathbb{Z}^{m}$. All our algorithms are simplex algorithms, meaning that they explicitly construct a path along the edges of $P$ from any given starting vertex $x^{0}$ to a vertex maximizing the liner function $c^{\top} x$. For this reason, we always assume that we are given a starting vertex $x^{0}$ of $P$. Obtaining an arbitrary starting vertex $x^{0}$ can be accomplished via Tardos' algorithm by performing a number of operations that is polynomially bounded in $\operatorname{size}(A)$. Recall that the size of the matrix $A$, denoted by $\operatorname{size}(A)$, is in $O(n m \log \alpha)$ (see Section 2.1 in [23] for more details).

The goal of this section is to present and analyze the preprocessing \& scaling algorithm. In order to do so, we first introduce the oracle, that is the basic building block of all our algorithms, and then the basic algorithm and the scaling algorithm.

\subsection{The oracle}

It will be convenient to consider the following oracle, which provides a general way to construct the next vertex in the simplex path.

Oracle

Input: A polytope $P$, a cost vector $c \in \mathbb{Z}^{n}$, and a vertex $x^{t}$ of $P$.

Output: Either a statement that $x^{t}$ is optimal (i.e., $x^{t} \in \operatorname{argmax}\left\{c^{\top} x \mid x \in P\right\}$ ), or a vertex $x^{t+1}$ adjacent to $x^{t}$ with strictly larger cost (i.e., $c^{\top} x^{t+1}>c^{\top} x^{t}$ ).

In the next remark we analyze the complexity of the oracle. We recall that a polytope $P=\left\{x \in \mathbb{R}^{n} \mid A x \leq b\right\}$ is said to be simple if, in each vertex of $P$, exactly $n$ inequalities from the system $A x \leq b$ are satisfied at equality.

Remark 1. An oracle call can be performed with a number of operations polynomially bounded in $\operatorname{size}(A)$. If $P$ is simple, it can be performed in $O(n m)$ operations. 
Proof. If the polytope $P$ is simple, then the oracle can be performed in $O(m n)$ operations. This can be seen using a pivot of the dual simplex method, where the primal is in standard form, and the feasible region of the dual is given by the polytope $P[5]$.

Consider now the case where $P$ may be not simple. Denote by $A^{=} x \leq b^{=}$the subsystem of the inequalities of $A x \leq b$ satisfied at equality by $x^{t}$. Note that the polyhedron $T:=$ $\left\{x \in \mathbb{R}^{n} \mid A^{=} x \leq b^{=}\right\}$is a translated cone with vertex $x^{t}$. Denote by $d^{\top}$ the sum of all the rows in $A^{=}$and note that the vertex $x^{t}$ is the unique maximizer of $d^{\top} x$ over $T$. Let $T^{\prime}$ be the truncated cone $T^{\prime}:=\left\{x \in T \mid d^{\top} x \geq d^{\top} x^{t}-1\right\}$ and note that there is a bijection between the neighbors of $x^{t}$ in $P$ and the vertices of $T^{\prime}$ different from $x^{t}$. We solve the LP problem $\max \left\{c^{\top} x \mid x \in T^{\prime}\right\}$. Using Tardos' algorithm, this LP can be solved in a number of operations that is polynomial in the size of the constraint matrix, which is polynomial in $\operatorname{size}(A)$.

If $x^{t}$ is an optimal solution to the LP, then the oracle returns that $x^{t}$ is optimal. Otherwise, Tardos' algorithm returns an optimal solution that is a vertex $w$ of $T^{\prime}$ different from $x^{t}$. In this case the oracle needs to return the corresponding neighbor $x^{t+1}$ of $x^{t}$ in $P$. Let $A^{\prime} x \leq b^{\prime}$ be the system obtained from $A x \leq b$ by setting to equality the inequalities in the subsystem $A^{=} x \leq b^{=}$satisfied at equality by both $x^{t}$ and $w$. It should be noted that the vectors that satisfy $A^{\prime} x \leq b^{\prime}$ constitute the edge of $P$ between $x^{t}$ and $x^{t+1}$. The vector $x^{t+1}$ can then be found by maximizing $c^{\top} x$ over $A^{\prime} x \leq b^{\prime}$ with Tardos' algorithm.

In all our algorithms, the simplex path is constructed via a number of oracle calls with different inputs. We note that, whenever $x^{t}$ is not optimal, our oracle has the freedom to return any adjacent vertex $x^{t+1}$ with strictly larger cost. Therefore, our algorithms can all be customized by further requiring the oracle to obey a specific pivoting rule.

\subsection{The basic algorithm}

The simplest way to solve (11) is to recursively invoke the oracle with input $P, c$, and the vertex $x^{t}$ obtained from the previous iteration, starting from the vertex $x^{0}$ in input. We formally describe this basic algorithm, which will be used as a subroutine in our subsequent algorithms.

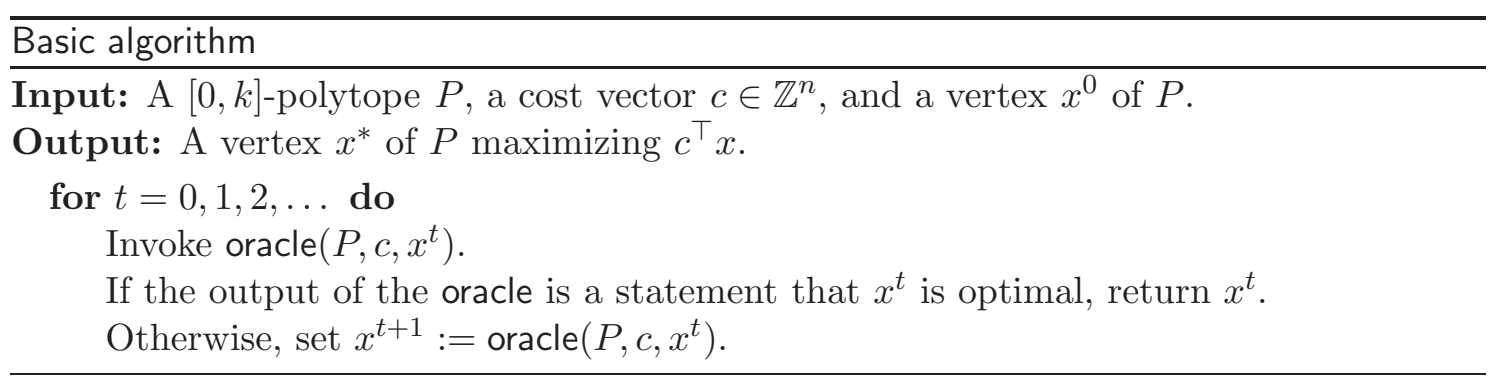

The correctness of the basic algorithm is immediate. Next, we upper bound the length of the simplex path generated by the basic algorithm.

Observation 1. The length of the simplex path generated by the basic algorithm is bounded by $c^{\top} x^{*}-c^{\top} x^{0}$. In particular, it is bounded by $n k\|c\|_{\infty}$. 
Proof. To show the first part of the statement, we only need to observe that each oracle call increases the objective value by at least one, since $c$ and the vertices of $P$ are integral.

The cost difference between $x^{*}$ and $x^{0}$ of $P$ can be bounded by

$$
c^{\top} x^{*}-c^{\top} x^{0}=\sum_{i=1}^{n} c_{i}\left(x_{i}^{*}-x_{i}^{0}\right) \leq \sum_{i=1}^{n}\left|c_{i}\right|\left|x_{i}^{*}-x_{i}^{0}\right| \leq n k\|c\|_{\infty},
$$

where the last inequality we use $\left|x_{i}^{*}-x_{i}^{0}\right| \leq k$ since $P$ is a $[0, k]$-polytope. This concludes the proof of the second part of the statement.

From Remark 1, we directly obtain the following.

Remark 2. The number of operations performed by the basic algorithm to construct the next vertex in the simplex path is polynomially bounded in size $(A)$. If $P$ is simple, the number of operations is $O(\mathrm{~nm})$.

\subsection{The scaling algorithm}

The length of the simplex path generated by the basic algorithm is clearly not satisfactory. In fact, as we discussed in Section 1, our goal is to obtain a simplex path of length polynomial in $n$ and $k$, and therefore independent from $\|c\|_{\infty}$. In this section we improve this gap by giving a scaling algorithm that yields a simplex path of length in $O\left(n k \log \|c\|_{\infty}\right)$.

Our scaling algorithm is based on a bit scaling technique. For ease of notation, we define $\ell:=\left\lceil\log \|c\|_{\infty}\right\rceil$. The main idea is to iteratively use the basic algorithm with the sequence of increasingly accurate integral approximations of the cost vector $c$ given by

$$
c^{t}:=\left\lceil\frac{c}{2^{\ell-t}}\right\rceil \quad \text { for } t=0, \ldots, \ell
$$

Since $c$ is an integral vector, we have $c^{\ell}=c$.

Bit scaling techniques have been extensively used to develop polynomial-time algorithms for a wide array of discrete optimization problems. Edmonds and Karp [13] and Dinic [12] independently introduced this technique in the context of the minimum cost flow problem. Gabow [15] used it for shortest path, maximum flow, assignment, and matching problems, while Schulz, Weismantel, and Ziegler 25] employed it to design a primal algorithm for 0/1-integer programming. The book [2] popularized bit scaling as a generic algorithmic tool in optimization.

Next, we describe our algorithm.

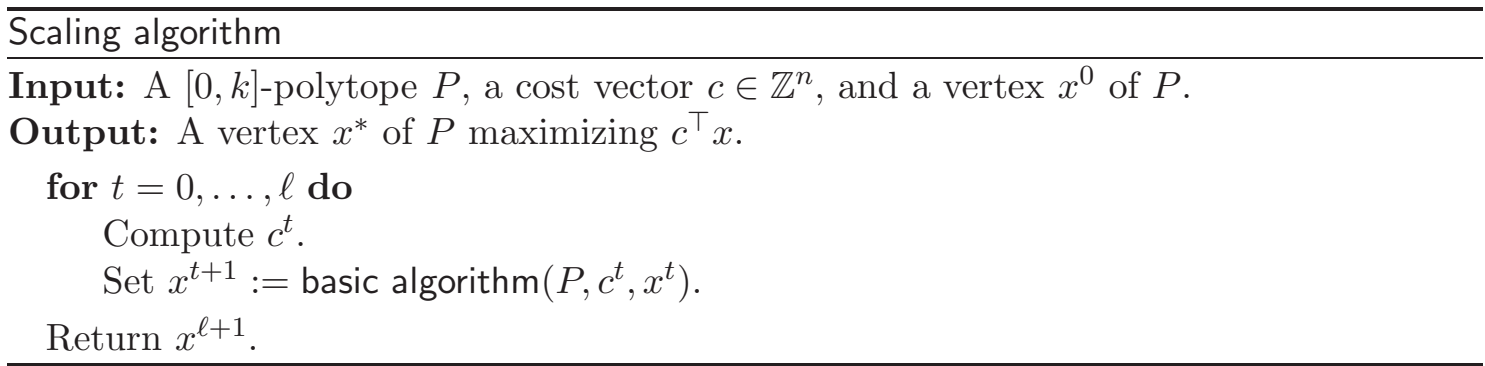


The correctness of the scaling algorithm follows from the correctness of the basic algorithm, since the vector $x^{\ell+1}$ returned is the output of the basic algorithm with input $P$ and cost vector $c^{\ell}=c$.

Next, we analyze the length of the simplex path generated by the scaling algorithm. We first derive some simple properties of the approximations $c^{t}$ of $c$.

Lemma 1. For each $t=0, \ldots, \ell$, we have $\left\|c^{t}\right\|_{\infty} \leq 2^{t}$.

Proof. By definition of $\ell$, we have $\left|c_{j}\right| \leq\|c\|_{\infty} \leq 2^{\ell}$ for every $j=1, \ldots, n$, hence $-2^{\ell} \leq$ $c_{j} \leq 2^{\ell}$. For any $t \in\{0, \ldots, \ell\}$, we divide the latter chain of inequalities by $2^{\ell-t}$ and round up to obtain

$$
-2^{t}=\left\lceil-2^{t}\right\rceil=\left\lceil\frac{-2^{\ell}}{2^{\ell-t}}\right\rceil \leq\left\lceil\frac{c_{j}}{2^{\ell-t}}\right\rceil \leq\left\lceil\frac{2^{\ell}}{2^{\ell-t}}\right\rceil=\left\lceil 2^{t}\right\rceil=2^{t} .
$$

Lemma 2. For each $t=1, \ldots, \ell$, we have $2 c^{t-1}-c^{t} \in\{0,1\}^{n}$.

Proof. First, we show that for every real number $r$, we have $2\lceil r\rceil-\lceil 2 r\rceil \in\{0,1\}$. Note that $r$ can be written as $\lceil r\rceil+f$ with $f \in(-1,0]$. We then have $\lceil 2 r\rceil=\lceil 2\lceil r\rceil+2 f\rceil=2\lceil r\rceil+$ $\lceil 2 f\rceil$. Since $\lceil 2 f\rceil \in\{-1,0\}$, we obtain $\lceil 2 r\rceil-2\lceil r\rceil \in\{-1,0\}$, hence $2\lceil r\rceil-\lceil 2 r\rceil \in\{0,1\}$.

Now, let $j \in\{1, \ldots, n\}$, and consider the $j$ th component of the vector $2 c^{t-1}-c^{t}$. By definition, we have

$$
2 c_{j}^{t-1}-c_{j}^{t}=2\left\lceil\frac{c_{j}}{2^{\ell-t+1}}\right\rceil-\left\lceil\frac{c_{j}}{2^{\ell-t}}\right\rceil .
$$

The statement then follows from the first part of the proof by setting $r=c_{j} / 2^{\ell-t+1}$.

We are ready to provide our bound on the length of the simplex path generated by the scaling algorithm. Even though the scaling algorithm uses the basic algorithm as a subroutine, we show that the simplex path generated by the scaling algorithm is much shorter than the one generated by the basic algorithm alone.

Proposition 1. The length of the simplex path generated by the scaling algorithm is bounded by $n k\left(\left\lceil\log \|c\|_{\infty}\right\rceil+1\right) \in O\left(n k \log \|c\|_{\infty}\right)$.

Proof. Note that the scaling algorithm performs a total number of $\ell+1=\left\lceil\log \|c\|_{\infty}\right\rceil+1$ iterations, and in each iteration it calls once the basic algorithm. Thus, we only need to show that, at each iteration, the simplex path generated by the basic algorithm is bounded by $n k$.

First we consider the iteration $t=0$ of the scaling algorithm. In this iteration, the basic algorithm is called with input $P, c^{0}$, and $x^{0}$. Lemma 1 implies that $\left\|c^{0}\right\|_{\infty} \leq 1$, and from Observation 1 we have that the basic algorithm calls the oracle at most $n k$ times.

Next, consider the iteration $t$ of the scaling algorithm for $t \in\{1, \ldots, \ell\}$. In this iteration, the basic algorithm is called with input $P, c^{t}$, and $x^{t}$, and outputs the vertex $x^{t+1}$. From Observation 1, we only need to show that $c^{t^{\top}} x^{t+1}-c^{t^{\top}} x^{t} \leq n k$. 
First, we derive an upper bound on $c^{t^{\top}} x^{t+1}$. By construction of $x^{t}$, the inequality $c^{t-1^{\top}} x \leq c^{t-1^{\top}} x^{t}$ is valid for the polytope $P$, thus

$$
c^{t^{\top}} x^{t+1}=\max \left\{c^{t^{\top}} x \mid x \in P\right\} \leq \max \left\{c^{t^{\top}} x \mid x \in[0, k]^{n}, c^{t-1^{\top}} x \leq c^{t-1^{\top}} x^{t}\right\} .
$$

The optimal value of the LP problem on the right-hand side is upper bounded by $2 c^{t-1^{\top}} x^{t}$. In fact, Lemma 2 implies $c^{t} \leq 2 c^{t-1}$, hence for every feasible vector $x$ of the LP problem on the right-hand side, we have

$$
c^{t^{\top}} x \leq 2 c^{t-1}{ }^{\top} x \leq 2 c^{t-1^{\top}} x^{t}
$$

Thus we have shown $c^{t^{\top}} x^{t+1} \leq 2 c^{t-1}{ }^{\top} x^{t}$.

We can now show $c^{t^{\top}} x^{t+1}-c^{t^{\top}} x^{t} \leq n k$. We have

$$
c^{t^{\top}} x^{t+1}-c^{t^{\top}} x^{t} \leq 2 c^{t-1}{ }^{\top} x^{t}-c^{t^{\top}} x^{t}=\left(2 c^{t-1}-c^{t}\right)^{\top} x^{t} \leq n k .
$$

The last inequality holds because, from Lemma 2, we know that $2 c^{t-1}-c^{t} \in\{0,1\}^{n}$, while the vector $x^{t}$ is in $[0, k]^{n}$.

Remark 3. The number of operations performed by the scaling algorithm to construct the next vertex in the simplex path is polynomially bounded in $\operatorname{size}(A)$ and $\log \|c\|_{\infty}$. If $P$ is simple, the number of operations is in $O\left(n^{2} m \log ^{2}\|c\|_{\infty}\right)$.

Proof. Let $x^{i}$ be the $i$-th vertex of the simplex path computed by the scaling algorithm. The scaling algorithm might call, in the worst case, $\ell$ times the basic algorithm before obtaining the next vertex $x^{i+1}$. Each time, the scaling algorithm first computes an approximation $c^{t}$ of $c$ and then calls the basic algorithm. Computing $c^{t}$ can be done by binary search, and the number of comparisons required is at most $n \log \left\|c^{t}\right\|_{\infty}$, which is bounded by $n t \leq n \ell$ from Lemma 1. Furthermore, from Remark 2, each time the basic algorithm is called, it performs a number of operations polynomially bounded in $\operatorname{size}(A)$, and by $O(\mathrm{~nm})$ if $P$ is simple. Therefore, to compute $x^{i+1}$ we need a number of operations bounded a polynomial in $\operatorname{size}(A)$ and in $\log \|c\|_{\infty}$. If $P$ is simple, the number of operations is $O(\ell \cdot n \ell \cdot n m)=O\left(n^{2} m \log ^{2}\|c\|_{\infty}\right)$.

In the next section we use the scaling algorithm as a subroutine in the preprocessing \& scaling algorithm. We remark that, the scaling algorithm will also be a subroutine in the iterative algorithm, which is described in Section 4 .

\subsection{The preprocessing \& scaling algorithm}

The length of the simplex path generated by the scaling algorithm still depends on $\|c\|_{\infty}$, even though the dependence is now logarithmic instead of linear. In this section we show that we can completely remove the dependence on $\|c\|_{\infty}$ by using our scaling algorithm in conjunction with the preprocessing algorithm by Frank and Tardos [14. This method relies on the simultaneous approximation algorithm of Lenstra, Lenstra and Lovász [19]. Next, we state the input and output of Frank and Tardos' algorithm. 
Preprocessing algorithm

Input: A vector $c \in \mathbb{Q}^{n}$ and a positive integer $N$.

Output: A vector $\breve{c} \in \mathbb{Z}^{n}$ such that $\|\breve{c}\|_{\infty} \leq 2^{4 n^{3}} N^{n(n+2)}$ and $\operatorname{sign}\left(c^{\top} z\right)=\operatorname{sign}\left(\breve{c}^{\top} z\right)$ for every $z \in \mathbb{Z}^{n}$ with $\|z\|_{1} \leq N-1$.

The number of operations performed by the preprocessing algorithm is polynomially bounded in $n$ and $\log N$. For more details, we refer the reader to Section 3 in 14 .

Next, we describe the algorithm obtained by combining the preprocessing algorithm and the scaling algorithm.

Preprocessing \& scaling algorithm

Input: $\mathrm{A}[0, k]$-polytope $P$, a cost vector $c \in \mathbb{Z}^{n}$, and a vertex $x^{0}$ of $P$.

Output: A vertex $x^{*}$ of $P$ maximizing $c^{\top} x$.

Set $\breve{c}:=$ preprocessing algorithm $(c, N:=n k+1)$.

Set $x^{*}:=\operatorname{scaling}$ algorithm $\left(P, \breve{c}, x^{0}\right)$.

Return $x^{*}$.

We first show that the preprocessing \& scaling algorithm is correct.

Proposition 2. The vertex $x^{*}$ of $P$ returned by the preprocessing \& scaling algorithm maximizes $c^{\top} x$ over $P$.

Proof. Due to the correctness of the scaling algorithm, we have that $\breve{c}^{\top}\left(x^{*}-x\right) \geq 0$ for every $x \in P$. Note that, for every $x \in P \cap \mathbb{Z}^{n}$, we have $x^{*}-x \in \mathbb{Z}^{n}$ and $\left\|x^{*}-x\right\|_{1} \leq n k=N-1$. Therefore, the preprocessing algorithm guarantees that $c^{\top}\left(x^{*}-x\right) \geq 0$ for every $x \in P \cap \mathbb{Z}^{n}$. The statement follows because all vertices of $P$ are integral.

We are now ready to give a proof of Theorem 1. We show that the obtained simplex path length is polynomially bounded in $n, k$, thus only polynomially far from the worstcase diameter.

Proof of Theorem [1. The vector $\breve{c}$ returned by the preprocessing algorithm satisfies $\|\breve{c}\|_{\infty} \leq$ $2^{4 n^{3}}(n k+1)^{n(n+2)}$, hence $\log \|\breve{c}\|_{\infty} \leq 4 n^{3} \log 2+n(n+2) \log (n k+1)$. From Proposition 1. the length of the simplex path generated by the preprocessing \& scaling algorithm is bounded by

$$
n k\left(\left\lceil\log \|\breve{c}\|_{\infty}\right\rceil+1\right) \leq n k\left(4 n^{3} \log 2+n(n+2) \log (n k+1)+2\right) \in O\left(n^{4} k \log (n k)\right) .
$$

We conclude this section by analyzing the number of operations performed by the preprocessing \& scaling algorithm.

Remark 4. The number of operations performed by the preprocessing \& scaling algorithm to construct the next vertex in the simplex path is polynomially bounded in $\operatorname{size}(A)$ and $\log k$. If $P$ is simple, the number of operations is polynomially bounded in $n, m$, and $\log k$. 
Proof. The number of operations performed by the preprocessing \& scaling algorithm to construct the next vertex in the simplex path is the sum of: (i) the number of operations needed to compute $\breve{c}$, and (ii) the number of operations performed by the scaling algorithm, with cost vector $\breve{c}$, to construct the next vertex in the simplex path. The vector $\breve{c}$ can be computed with a number of operations polynomially bounded in $n$ and $\log (n k)$ [14. From Remark 3, (ii) is polynomially bounded in $\operatorname{size}(A)$ and $\log \|\breve{c}\|_{\infty}$, and by $O\left(n^{2} m \log ^{2}\|\breve{c}\|_{\infty}\right)$ if $P$ is simple. To conclude the proof, we only need to observe that $\log \|\breve{c}\|_{\infty}$ is polynomially bounded in $n$ and $\log k$.

\section{The iterative algorithm}

In this section, we design a simplex algorithm that yields simplex path whose length depends on $n, k$, and $\alpha$, where $\alpha$ denotes the largest absolute value of the entries of $A$. We define $[m]:=\{1,2, \ldots, m\}$ and refer to the rows of $A$ as $a_{i}$, for $i \in[m]$. Next, we present our iterative algorithm.

Iterative algorithm

Input: $\mathrm{A}[0, k]$-polytope $P$, a cost vector $c \in \mathbb{Z}^{n}$, and a vertex $x^{0}$ of $P$.

Output: A vertex $x^{*}$ of $P$ maximizing $c^{\top} x$.

0 : Let $\mathcal{E}:=\emptyset$ and $x^{*}:=x^{0}$.

1: Let $\bar{c}$ be the projection of $c$ onto the subspace $\left\{x \in \mathbb{R}^{n} \mid a_{i}^{\top} x=0\right.$ for $\left.i \in \mathcal{E}\right\}$ of $\mathbb{R}^{n}$. If $\bar{c}=0$ return $x^{*}$, otherwise go to 2 ,

2: Let $\tilde{c} \in \mathbb{Z}^{n}$ be defined by $\tilde{c}_{i}:=\left\lfloor\frac{n^{3} k \alpha}{\|\bar{c}\|_{\infty}} \bar{c}_{i}\right\rfloor$ for $i=1, \ldots, n$.

3: Consider the following pair of primal and dual LP problems:

$$
\begin{array}{llllll}
\max & \tilde{c}^{\top} x & (\tilde{P}) & & \min & b^{\top} y \\
\text { s.t. } & a_{i}^{\top} x=b_{i} \quad i \in \mathcal{E} & \text { s.t. } & A^{\top} y=\tilde{c} & \\
& a_{i}^{\top} x \leq b_{i} \quad i \in[m] \backslash \mathcal{E} & & & y_{i} \geq 0 & i \in[m] \backslash \mathcal{E} .
\end{array}
$$

Use the scaling algorithm to compute an optimal vertex $\tilde{x}$ of $(\tilde{P})$ starting from $x^{*}$.

Compute an optimal solution $\tilde{y}$ to the dual $(\tilde{D})$ such that (i) $\tilde{y}$ has at most $n$ nonzero components, and (ii) $\tilde{y}_{j}=0$ for every $j \in[m] \backslash \mathcal{E}$ such that $a_{j}$ can be written as a linear combination of $a_{i}, i \in \mathcal{E}$.

Let $\mathcal{H}:=\left\{i \mid \tilde{y}_{i}>n k\right\}$, and let $h \in \mathcal{H} \backslash \mathcal{E}$. Add the index $h$ to the set $\mathcal{E}$, set $x^{*}:=\tilde{x}$, and go back to step 1 .

As the name suggests, the above algorithm is iterative in nature. In particular, an iteration of the algorithm corresponds to one execution of steps 1, 2, and 3 ,

\subsection{Well-defined}

In this section we prove that the iterative algorithm is well-defined, meaning that it can indeed perform all the instructions stated in its steps. First we show that, when the iterative algorithm calls the scaling algorithm in step 3, $x^{*}$ is a valid input. 
Lemma 3. In step 3, the iterative algorithm calls the scaling algorithm with valid inputs.

Proof. We only need to show that the vector $x^{*}$ is feasible for problem $(\tilde{P})$. Consider the vectors $\tilde{x}, \tilde{y}$ and the index $h$ from the previous iteration. We have $\tilde{y}_{h}>0$, which, by complementary slackness, implies $a_{h}^{\top} \tilde{x}=b_{h}$. Therefore, the $\tilde{x}$ from the previous iteration, which coincides with $x^{*}$ of the current iteration, is indeed feasible for the problem $(\tilde{P})$ of the current iteration.

Next, we show that an optimal solution $\tilde{y}$ to the dual $(\tilde{D})$ with the properties stated in step 3 exists, and it can be computed efficiently.

Lemma 4. In step 3 of the iterative algorithm, a vector $\tilde{y}$ satisfying (i) and (ii) always exists, and the number of operations needed to compute it is polynomially bounded in $\operatorname{size}(A)$. If $P$ is simple, the number of operations is in $O\left(n m+n^{3}\right)$.

Proof. First, assume that the polytope $P$ is simple. Let problem $(\tilde{\tilde{P}}$, be obtained from $(\tilde{P})$ by dropping the inequalities that are not active at $\tilde{x}$. This can be done in $O(n m)$ operations. Since $P$ is simple, the number of constraints in $(\underline{P})$, is $n$ and the $n \times n$ constraint matrix $\tilde{A}$ of $(\tilde{P})$ ' is invertible. Note that $\tilde{x}$ is an optimal solution to ( $\tilde{P}$ )' as well. Let $(\tilde{D})$ ' be the dual of $(\underline{\tilde{P}})$ '. Note that $(\tilde{D})$ ' is obtained from $(\tilde{D})$ by dropping the variables $y_{j}$ corresponding to the inequalites of $(\underline{\tilde{P}})$ dropped to obtain $(\underline{\tilde{P}})$ '. Since $(\underline{\tilde{P}})$ ' has an optimal solution, then so does $(\underline{\tilde{D}})$ ' from strong duality. The constraint matrix of ( $(\tilde{D})$, is the invertible matrix $\tilde{A}^{\top}$. The only feasible solution to the system of linear equations in $(\tilde{D})$ ' is the vector $\tilde{y}^{\prime}:=\tilde{A}^{-\top} \tilde{c}$ which can be computed in $O\left(n^{3}\right)$ operations. Since ( $\tilde{D}$, is feasible, then $\tilde{y}^{\prime}$ must satisfies all the constraints in $(\tilde{D})$, thus $\tilde{y}^{\prime}$ is optimal. Let $\tilde{y}$ be obtained from $\tilde{y}^{\prime}$ by adding back the dropped components and setting them to zero. The vector $\tilde{y}$ is feasible to $(\tilde{D})$, and, from complementary slackness with $\tilde{x}$, it is optimal to $(\tilde{D})$. Furthermore, $\tilde{y}$ clearly satisfies (i). To see that it satisfies (ii), note that the inequalities $a_{i}^{\top} x=b_{i}, i \in \mathcal{E}$, are all in $(\tilde{\tilde{P}})$. Since the constraints in $(\underline{\tilde{P}})$, are all linearly independent, problem $(\tilde{P})$ ' cannot contain any constraint $a_{j}^{\top} x \leq b_{j}$, for $j \in[m] \backslash \mathcal{E}$ such that $a_{j}$ can be written as a linear combination of $a_{i}, i \in \mathcal{E}$. Hence, the corresponding dual variable $\tilde{y}_{j}$ has been set to zero.

Consider now the general case where $P$ may be not simple. First, we show how to compute a vector $\tilde{y}$ that satisfies (i). Since $(\tilde{P})$ has an optimal solution, then so does $(\tilde{D})$ from strong duality. Let $(\underline{D})$ ' be obtained from $(\tilde{D})$ by replacing each variable $y_{i}, i \in \mathcal{E}$, with $y_{i}^{+}-y_{i}^{-}$, where $y_{i}^{+}$and $y_{i}^{-}$are new variables which are required to be nonnegative. Clearly $(\tilde{D})$ and $(\tilde{D})$ ' are equivalent, so $(\underline{\tilde{D}})$ ' has an optimal solution. Furthermore, since $(\tilde{D})$ ' is in standard form, it has an optimal solution $\tilde{y}^{\prime}$ that is a basic feasible solution. In particular, via Tardos' algorithm, the vector $\tilde{y}^{\prime}$ can be computed in a number of operations polynomially bounded in size $(A)$. Let $\tilde{y}$ be obtained from $\tilde{y}^{\prime}$ by replacing each pair $\tilde{y}_{i}^{\prime+}, \tilde{y}_{i}^{\prime-}$ with $\tilde{y}_{i}:=\tilde{y}_{i}^{\prime+}-\tilde{y}_{i}^{\prime-}$. It is simple to check that $\tilde{y}$ is an optimal solution to $(\tilde{D})$. Since $\tilde{y}^{\prime}$ is a basic feasible solution, it has at most $n$ nonzero entries. By construction, so does $\tilde{y}$.

Next, we discuss how to compute a vector $\tilde{y}$ that satisfies (i) and (ii). Let problem

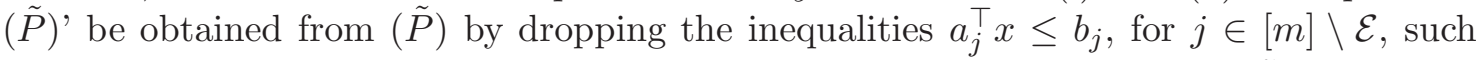
that $a_{j}$ can be written as a linear combination of $a_{i}, i \in \mathcal{E}$. Since problem $(\underline{\tilde{P}})$ is feasible, then $(\tilde{P})$ and $(\underline{\tilde{P}})$, have the same feasible region and are therefore equivalent. Let $(\underline{\tilde{D}})$, 
be the dual of $(\underline{\tilde{P}})$ '. Note that $(\underline{\tilde{D}})$ ' is obtained from $(\underline{\tilde{D}})$ by dropping the variables $y_{j}$ corresponding to the inequalities of $(\underline{\tilde{P}})$ dropped to obtain $(\underline{\tilde{P}})$. . Note that $(\underline{\tilde{P}})$ ' has the same form of $(\tilde{P})$, thus, from the the first part of the proof, we can compute a vector $\tilde{y}^{\prime}$ optimal to $(\tilde{D})$ ' with at most $n$ nonzero components. Furthermore, $\tilde{y}^{\prime}$ can be computed in a number of operations polynomially bounded in size $(A)$. Let $\tilde{y}$ be obtained from $\tilde{y}^{\prime}$ by adding back the dropped components and setting them to zero. The vector $\tilde{y}$ is feasible to $(\tilde{D})$, and, from complementary slackness with $\tilde{x}$, it is optimal to $(\tilde{D})$. Furthermore, $\tilde{y}$ satisfies (i) and (ii).

In the next lemma, we show that at step 3 we can always find an index $h \in \mathcal{H} \backslash \mathcal{E}$.

Lemma 5. In step 3 of the iterative algorithm, we have $\mathcal{H} \backslash \mathcal{E} \neq \emptyset$. In particular, the index $h$ exists at each iteration.

Proof. Let $\bar{c}, \tilde{c}, \tilde{x}$ and $\tilde{y}$ be the vectors computed at a generic iteration of the iterative algorithm. Let $\hat{c}=\frac{n^{3} k \alpha}{\|\bar{c}\|_{\infty}} \bar{c}$, and note that $\tilde{c}=\lfloor\hat{c}\rfloor$. Moreover, we have $\|\hat{c}\|_{\infty}=n^{3} k \alpha$ and, since this number is integer, we also have $\|\tilde{c}\|_{\infty}=n^{3} k \alpha$.

Let $\mathcal{B}=\left\{i \in\{1, \ldots, m\} \mid \tilde{y}_{i} \neq 0\right\}$. From property (i) of the vector $\tilde{y}$ we know $|\mathcal{B}| \leq n$. From the constraints of $(\tilde{D})$ we obtain

$$
\tilde{c}=\sum_{i \in[m]} a_{i} \tilde{y}_{i}=\sum_{i \in \mathcal{B}} a_{i} \tilde{y}_{i}
$$

Note that $\tilde{y}_{j} \geq 0$ for every $j \in \mathcal{B} \backslash \mathcal{E}$ since $\tilde{y}$ is feasible to $(\tilde{D})$. Hence to prove this lemma we only need to show that

$$
\left|\tilde{y}_{j}\right|>n k \quad \text { for some } j \in \mathcal{B} \backslash \mathcal{E} .
$$

The proof of (4) is divided into two cases.

In the first case we assume $\mathcal{B} \cap \mathcal{E}=\emptyset$. Thus, to prove (4), we only need to show that $\left|\tilde{y}_{j}\right|>n k$ for some $j \in \mathcal{B}$. To obtain a contradiction, we suppose $\left|\tilde{y}_{j}\right| \leq n k$ for every $j \in \mathcal{B}$. From (3) we obtain

$$
\|\tilde{c}\|_{\infty} \leq \sum_{j \in \mathcal{B}}\left\|a_{j} \tilde{y}_{j}\right\|_{\infty}=\sum_{j \in \mathcal{B}}\left(\left|\tilde{y}_{j}\right|\left\|a_{j}\right\|_{\infty}\right) \leq \sum_{j \in \mathcal{B}}(n k \cdot \alpha) \leq n^{2} k \alpha
$$

However, this contradicts the fact that $\|\tilde{c}\|_{\infty}=n^{3} k \alpha$. Thus $\left|\tilde{y}_{j}\right|>n k$ for some $j \in \mathcal{B}$, and (44) holds. This concludes the proof in the first case.

In the second case we assume that $\mathcal{B} \cap \mathcal{E}$ is nonempty. In particular, we have $|\mathcal{B} \backslash \mathcal{E}| \leq$ $n-1$. In order to derive a contradiction, suppose that (4) does not hold, i.e., $\left|\tilde{y}_{j}\right| \leq n k$ for every $j \in \mathcal{B} \backslash \mathcal{E}$. From (3) we obtain

$$
\tilde{c}=\sum_{i \in \mathcal{B}} a_{i} \tilde{y}_{i}=\sum_{i \in \mathcal{B} \cap \mathcal{E}} a_{i} \tilde{y}_{i}+\sum_{j \in \mathcal{B} \backslash \mathcal{E}} a_{j} \tilde{y}_{j} .
$$


Then

$$
\begin{aligned}
\left\|\tilde{c}-\sum_{i \in \mathcal{B} \cap \mathcal{E}} a_{i} \tilde{y}_{i}\right\|_{\infty} & \leq \sum_{j \in \mathcal{B} \backslash \mathcal{E}}\left\|a_{j} \tilde{y}_{j}\right\|_{\infty}=\sum_{j \in \mathcal{B} \backslash \mathcal{E}}\left(\left|\tilde{y}_{j}\right|\left\|a_{j}\right\|_{\infty}\right) \\
& \leq \sum_{j \in \mathcal{B} \backslash \mathcal{E}}(n k \cdot \alpha) \leq(n-1) n k \alpha \leq n^{2} k \alpha-1 .
\end{aligned}
$$

Next, in order to derive a contradiction, we show that

$$
\left\|\tilde{c}-\sum_{i \in \mathcal{B} \cap \mathcal{E}} a_{i} \tilde{y}_{i}\right\|_{\infty}>n^{2} k \alpha-1
$$

By adding and removing $\tilde{c}$ inside the norm in the left-hand side below, we obtain

$$
\left\|\hat{c}-\sum_{i \in \mathcal{B} \cap \mathcal{E}} a_{i} \tilde{y}_{i}\right\|_{\infty}=\left\|\tilde{c}-\sum_{i \in \mathcal{B} \cap \mathcal{E}} a_{i} \tilde{y}_{i}-(\tilde{c}-\hat{c})\right\|_{\infty} \leq\left\|\tilde{c}-\sum_{i \in \mathcal{B} \cap \mathcal{E}} a_{i} \tilde{y}_{i}\right\|_{\infty}+\|\tilde{c}-\hat{c}\|_{\infty} .
$$

Let us now focus on the left-hand side of (77). We have that $\hat{c}$ is orthogonal to $a_{i}$, for every $i \in \mathcal{E}$. This is because $\hat{c}$ is a scaling of $\bar{c}$ and the latter vector is, by definition, orthogonal to $a_{i}$, for every $i \in \mathcal{E}$. We obtain

$$
\left\|\hat{c}-\sum_{i \in \mathcal{B} \cap \mathcal{E}} a_{i} \tilde{y}_{i}\right\|_{\infty} \geq \frac{1}{\sqrt{n}}\left\|\hat{c}-\sum_{i \in \mathcal{B} \cap \mathcal{E}} a_{i} \tilde{y}_{i}\right\|_{2} \geq \frac{\|\hat{c}\|_{2}}{\sqrt{n}} \geq \frac{\|\hat{c}\|_{\infty}}{\sqrt{n}}=\frac{n^{3} k \alpha}{\sqrt{n}} \geq n^{2} k \alpha,
$$

where the second inequality holds by Pythagoras' theorem.

Using (77), (8), and noting that $\|\tilde{c}-\hat{c}\|_{\infty}<1$ by definition of $\tilde{c}$, we obtain

$$
\left\|\tilde{c}-\sum_{i \in \mathcal{B} \cap \mathcal{E}} a_{i} \tilde{y}_{i}\right\|_{\infty} \geq\left\|\hat{c}-\sum_{i \in \mathcal{B} \cap \mathcal{E}} a_{i} \tilde{y}_{i}\right\|_{\infty}-\|\tilde{c}-\hat{c}\|_{\infty}>n^{2} k \alpha-1 .
$$

This concludes the proof of (6) .

Inequalities (5) and (6) yield a contradiction, thus (4) holds. This concludes the proof in the second case.

\subsection{Correctness}

Our next goal is to prove that the iterative algorithm is correct, i.e., that it returns a vertex of $P$ maximizing $c^{\top} x$.

At each iteration of the iterative algorithm, let $F$ be the face of $P$ defined as

$$
F:=\left\{x \in \mathbb{R}^{n} \mid a_{i}^{\top} x \leq b_{i} \text { for } i \in[m] \backslash \mathcal{E}, a_{i}^{\top} x=b_{i} \text { for } i \in \mathcal{E}\right\},
$$

and note that $F$ is the feasible region of $(\underline{\tilde{P}})$. The next lemma implies that at each iteration the dimension of $F$ decreases by 1 . 
Lemma 6. At each iteration, the row submatrix of $A$ indexed by $\mathcal{E}$ has full row rank. Furthermore, at each iteration, its number of rows increases by exactly one.

Proof. We prove this lemma recursively. Clearly, the statement holds at the beginning of the algorithm because we have $\mathcal{E}=\emptyset$.

Assume now that, at a general iteration, the row submatrix of $A$ indexed by $\mathcal{E}$ has full row rank. From Lemma 5, the index $h \in \mathcal{H} \backslash \mathcal{E}$ defined in step 3 of the algorithm exists. In particular, $\tilde{y}_{h}>n k$. From property (ii) of the vector $\tilde{y}$, we have that $a_{h}$ is linearly independent from the vectors $a_{i}, i \in \mathcal{E}$. Hence the rank of the row submatrix of $A$ indexed by $\mathcal{E} \cup\{h\}$ is one more than the rank of the row submatrix of $A$ indexed by $\mathcal{E}$. In particular, it has full row rank.

In the next three lemmas, we will prove that, at each iteration, every optimal solution to (11) lies in $F$. Note that, since $F$ is a face of $P$, it is also a $[0, k]$-polytope.

Suppose that an optimal solution $\tilde{y}$ of $(\tilde{D})$ is known. The complementary slackness conditions for linear programming imply that, for every $\tilde{x}$ optimal for $(\tilde{P})$, we have

$$
\tilde{y}_{i}>0 \Rightarrow a_{i}^{\top} \tilde{x}=b_{i} \quad i \in[m] \backslash \mathcal{E} .
$$

Thus, in order to solve $(\underline{\tilde{P}})$, we can restrict the feasible region of $(\underline{\tilde{P}})$ by setting the primal constraints in (9) to equality.

Now, suppose that our goal is to solve a variant of $(\tilde{P})$ where we replace the cost vector $\tilde{c}$ with another cost vector $\hat{c}$. Denote by $(\hat{P})$ this new LP problem. Note that $\tilde{y}$ might not even be feasible for the dual problem $(\hat{D})$ associated to $(\hat{P})$. Can we, under suitable conditions, still use $\tilde{y}$ to conclude that a primal constraint is satisfied with equality by each optimal solution to $(\hat{P})$ ? We show that, if $\tilde{y}$ is 'close' to being feasible for $(\hat{D})$, then for each index $i \in[m] \backslash \mathcal{E}$ such that $\tilde{y}_{i}$ is sufficiently large, we have that the corresponding primal constraint is active at every optimal solution to $(\hat{P})$. Thus, in order to solve $(\hat{P})$, we can restrict the feasible region of $(\hat{P})$ by setting these primal constraints to equality.

In the following, for $u \in \mathbb{R}^{n}$ we denote by $|u|$ the vector whose entries are $\left|u_{i}\right|, i=$ $1, \ldots n$.

Lemma 7. Let $\tilde{x} \in F, \hat{c} \in \mathbb{R}^{n}$, and $\tilde{y} \in \mathbb{R}^{m}$ be such that

$$
\begin{array}{ll}
\left|A^{\top} \tilde{y}-\hat{c}\right| \leq 1 & \\
\tilde{y}_{i} \geq 0 & i \in[m] \backslash \mathcal{E} \\
\tilde{y}_{i}>0 \Rightarrow a_{i}^{\top} \tilde{x}=b_{i} & i \in[m] \backslash \mathcal{E} .
\end{array}
$$

Then for any vector $\hat{x} \in F \cap \mathbb{Z}^{n}$ with $\hat{c}^{\top} \hat{x} \geq \hat{c}^{\top} \tilde{x}$, we have

$$
\tilde{y}_{i}>n k \Rightarrow a_{i}^{\top} \hat{x}=b_{i} \quad i \in[m] \backslash \mathcal{E} .
$$

Proof. Let $u:=(\hat{x}-\tilde{x})$, and let $u^{+}, u^{-} \in \mathbb{R}_{+}^{n}$ be defined as follows. For $j \in[n]$,

$$
u_{j}^{+}:=\left\{\begin{array}{ll}
u_{j} & \text { if } u_{j} \geq 0 \\
0 & \text { if } u_{j}<0,
\end{array} \quad u_{j}^{-}:= \begin{cases}0 & \text { if } u_{j} \geq 0 \\
-u_{j} & \text { if } u_{j}<0\end{cases}\right.
$$


Clearly $u=u^{+}-u^{-}$and $|u|=u^{+}+u^{-}$. Since $\hat{c}^{\top} \hat{x} \geq \hat{c}^{\top} \tilde{x}$, we have $\hat{c}^{\top} u \geq 0$.

We prove this lemma by contradiction. Suppose that there exists $h \in[m] \backslash \mathcal{E}$ such that $\tilde{y}_{h}>n k$ and $a_{h}^{\top} \hat{x} \neq b_{h}$. Since $\hat{x} \in F$ and $a_{h}, \hat{x}, b_{h}$ are integral, we have $a_{h}^{\top} \hat{x} \leq b_{h}-1$. We rewrite (10) as $A^{\top} \tilde{y}-1 \leq \hat{c} \leq A^{\top} \tilde{y}+1$. Thus

$$
\begin{aligned}
\hat{c}^{\top} u & =\hat{c}^{\top} u^{+}-\hat{c}^{\top} u^{-} \leq\left(A^{\top} \tilde{y}+1\right)^{\top} u^{+}-\left(A^{\top} \tilde{y}-1\right)^{\top} u^{-} \\
& =\left(A^{\top} \tilde{y}\right)^{\top}\left(u^{+}-u^{-}\right)+1^{\top}\left(u^{+}+u^{-}\right)=\left(A^{\top} \tilde{y}\right)^{\top} u+1^{\top}|u| .
\end{aligned}
$$

We can upper bound $1^{\top}|u|$ in (13) by observing that $\left|u_{j}\right| \leq k$ for all $j \in[n]$, since $u$ is the difference of two vectors in $[0, k]^{n}$. Thus

$$
1^{\top}|u| \leq n k
$$

We now compute an upper bound for $\left(A^{\top} \tilde{y}\right)^{\top} u=\tilde{y}^{\top} A u$ in (13).

$$
\begin{aligned}
\tilde{y}^{\top} A u & =\tilde{y}_{h} a_{h}^{\top} u+\sum_{i \in \mathcal{E}} \tilde{y}_{i} a_{i}^{\top} u+\sum_{i \in[m] \backslash \mathcal{E}, i \neq h} \tilde{y}_{i} a_{i}^{\top} u \\
& <-n k+\sum_{i \in \mathcal{E}} \tilde{y}_{i} a_{i}^{\top} u+\sum_{i \in[m] \backslash \mathcal{E}, i \neq h} \tilde{y}_{i} a_{i}^{\top} u \\
& =-n k+\sum_{i \in[m] \backslash \mathcal{E}, i \neq h} \tilde{y}_{i} a_{i}^{\top} u \\
& \leq-n k+\sum_{i \in[m] \backslash \mathcal{E}, i \neq h, \tilde{y}_{i}>0} \tilde{y}_{i} a_{i}^{\top} u \\
& \leq-n k .
\end{aligned}
$$

To prove the strict inequality in (15) we show $\tilde{y}_{h} a_{h}^{\top} u<-n k$. We have $\tilde{y}_{h}>n k>0$, thus condition (12) implies $a_{h}^{\top} \tilde{x}=b_{h}$. Since $a_{h}^{\top} \hat{x} \leq b_{h}-1$, we get $a_{h}^{\top} u=a_{h}^{\top} \hat{x}-a_{h}^{\top} \tilde{x} \leq-1$. We multiply $\tilde{y}_{h}>n k$ by $a_{h}^{\top} u$ and obtain $\tilde{y}_{h} \cdot a_{h}^{\top} u<n k \cdot a_{h}^{\top} u \leq-n k$. Equality (16) follows from the fact that, for each $i \in \mathcal{E}$ we have $a_{i}^{\top} \hat{x}=b_{i}$ and $a_{i}^{\top} \tilde{x}=b_{i}$ since both $\hat{x}$ and $\tilde{x}$ are in $F$, thus $a_{i}^{\top} u=0$. Inequality (17) follows from (11). To see why inequality (18) holds, first note that, from condition (12), $\tilde{y}_{i}>0$ implies $a_{i}^{\top} \tilde{x}=b_{i}$. Furthermore, since $\hat{x} \in F$, we have $a_{i}^{\top} \hat{x} \leq b_{i}$. Hence we have $a_{i}^{\top} u \leq 0$ and so $\tilde{y}_{i} a_{i}^{\top} u \leq 0$.

By combining (13), (14) and (18) we obtain $\hat{c}^{\top} u<0$. This is a contradiction since we have previously seen that $\hat{c}^{\top} u \geq 0$.

For a vector $w \in \mathbb{Z}^{n}$ and a polyhedron $Q \subseteq \mathbb{R}^{n}$, we say that a vector is $w$-maximal in $Q$ if it maximizes $w^{\top} x$ over $Q$.

Lemma 8. The set $\mathcal{H}$ given at step 3 of the iterative algorithm is such that every vector $\hat{x}$ that is $\bar{c}$-maximal in $F$ satisfies $a_{i}^{\top} \hat{x}=b_{i}$ for every $i \in \mathcal{H}$.

Proof. Clearly, we just need to prove the lemma for every vertex $\hat{x}$ of $F$ that maximizes $\bar{c}^{\top} x$ over $F$. In particular, $\hat{x}$ is a vertex of $P$ and is therefore integral. 
Define $\hat{c} \in \mathbb{R}^{n}$ as $\hat{c}_{i}:=\frac{n^{3} k \alpha}{\|\bar{c}\|_{\infty}} \bar{c}_{i}$ for $i=1, \ldots, n$. At step 3, $\tilde{x}$ is an optimal vertex of $(\underline{\tilde{P}})$, and $\tilde{y}$ is an optimal solution to the dual $(\tilde{D})$. We have:

$$
\begin{aligned}
& \left|A^{\top} \tilde{y}-\hat{c}\right| \leq 1 \\
& \tilde{y}_{i} \geq 0 \quad i \in[m] \backslash \mathcal{E} .
\end{aligned}
$$

Constraints (20) are satisfied since $\tilde{y}$ is feasible for ( $(\tilde{D})$. Condition (19) holds because $\left|A^{\top} \tilde{y}-\hat{c}\right|=|\tilde{c}-\hat{c}|=\hat{c}-\tilde{c}<1$. Moreover, the complementary slackness conditions (9) are satisfied by $\tilde{x}$ and $\tilde{y}$, because they are optimal for $(\tilde{\tilde{P}})$ and $(\tilde{D})$, respectively.

Thus, $A, b, \hat{c}, \tilde{x}, \tilde{y}$ satisfy the hypotheses of Lemma 7 . Since the vector $\hat{x}$ is $\bar{c}$-maximal in $F$ and $\hat{c}$ is a scaling of $\bar{c}$, the vector $\hat{x}$ is also $\hat{c}$-maximal in $F$. Since $\tilde{x} \in F$, we have $\hat{c}^{\top} \hat{x} \geq \hat{c}^{\top} \tilde{x}$. Then Lemma 7 implies

$$
\tilde{y}_{i}>n k \Rightarrow a_{i}^{\top} \hat{x}=b_{i} \quad i \in[m] \backslash \mathcal{E},
$$

that is, $a_{i}^{\top} \hat{x}=b_{i}$ for all $i \in \mathcal{H}$.

Lemma 9. The set $\mathcal{E}$ updated in step 3 of the iterative algorithm is such that every vector $x^{*}$ that is c-maximal in $P$ satisfies $a_{i}^{\top} x^{*}=b_{i}$ for $i \in \mathcal{E}$.

Proof. Consider a vector $x^{*}$ that is $c$-maximal in $P$. We prove this lemma recursively. Clearly, the statement is true at the beginning of the algorithm, when $\mathcal{E}=\emptyset$.

Suppose now that the statement is true at the beginning of a general iteration. At the beginning of step 3 we have that $x^{*}$ is $c$-maximal in $F$, thus it is also $\bar{c}$-maximal in $F$. When we add an index $h \in \mathcal{H} \backslash \mathcal{E}$ to $\mathcal{E}$ at the end of step 3, by Lemma 8 we obtain that $a_{h}^{\top} x^{*}=b_{h}$. Thus, at each iteration of the algorithm we have $a_{i}^{\top} x^{*}=b_{i}$ for $i \in \mathcal{E}$.

In the next theorem, we show that the iterative algorithm is correct.

Theorem 3. The vector $x^{*}$ returned by the iterative algorithm is an optimal solution to the LP problem (11).

Proof. Consider the iteration of the iterative algorithm when the vector $x^{*}$ is returned at step 1. Let the set $\mathcal{E}$ be as defined in the algorithm when $x^{*}$ is returned. Up to reordering the inequalities defining $P$, we can assume, without loss of generality, that $\mathcal{E}=\{1, \ldots, r\}$. Consider the following primal/dual pair:

$$
\begin{aligned}
& \max c^{\top} x \quad \min b^{\top} y \\
& \begin{array}{ll}
\text { s.t. } & a_{i}^{\top} x=b_{i} \quad i=1, \ldots, r \\
& a_{i}^{\top} x \leq b_{i} \quad i=r+1, \ldots, m
\end{array} \\
& \text { s.t. } \quad A^{\top} y=c \\
& y_{i} \geq 0 \quad i=r+1, \ldots, m \text {. }
\end{aligned}
$$

Note that the feasible region of $(\underline{P})$ is the same of $(\tilde{P})$, and it consists of the face $F$ of $P$ obtained by setting to equality all the constraints indexed by $\mathcal{E}$. Furthermore, the objective function of $(P)$ coincides with the one of (10).

Let $A_{\mathcal{E}}$ be the row submatrix of $A$ indexed by $\mathcal{E}$. By Lemma 6 , the rank of $A_{\mathcal{E}}$ is $r$. When, at step 1, we project $c$ onto $\left\{x \in \mathbb{R}^{n} \mid A_{\mathcal{E}} x=0\right\}$, we get $\bar{c}=c-A_{\mathcal{E}}^{\top}\left(A_{\mathcal{E}} A_{\mathcal{E}}^{\top}\right)^{-1} A_{\mathcal{E}} c$. 
Since the termination condition is triggered at this iteration, we have $\bar{c}=0$. This implies $c=A_{\mathcal{E}}^{\top} z$, where $z:=\left(A_{\mathcal{E}} A_{\mathcal{E}}^{\top}\right)^{-1} A_{\mathcal{E}} c$.

Let $\bar{y} \in \mathbb{R}^{m}$ be defined by $\bar{y}_{i}:=z_{i}$ for $i=1, \ldots, r$, and $\bar{y}_{i}:=0$ for $i=r+1, \ldots, m$. First, $\bar{y}$ is feasible for (D). In fact $\bar{y}_{i} \geq 0$ for $i=r+1, \ldots, m$ and

$$
A^{\top} \bar{y}=\sum_{i=1}^{m} \bar{y}_{i} a_{i}=\sum_{i=1}^{r} z_{i} a_{i}=A_{\mathcal{E}}^{\top} z=c .
$$

In particular, $x^{*}$ is feasible for $(P)$. We have

$$
c^{\top} x^{*}=\bar{y}^{\top} A x^{*}=\sum_{i=1}^{m} \bar{y}_{i} a_{i}^{\top} x^{*}=\sum_{i=1}^{r} \bar{y}_{i} a_{i}^{\top} x^{*}=\sum_{i=1}^{r} \bar{y}_{i} b_{i}=\bar{y}^{\top} b .
$$

By strong duality, $x^{*}$ is $c$-maximal in $F$. If $x^{*}$ is not $c$-maximal in $P$, then there exist a different vector $x^{\dagger}$ that is $c$-maximal in $P$. In particular, we have $c^{\top} x^{\dagger}>c^{\top} x^{*}$. From Lemma 9, the vector $x^{\dagger}$ lies in $F$. Since $x^{*}$ is $c$-maximal in $F$, we obtain $c^{\top} x^{\dagger} \leq c^{\top} x^{*}$, a contradiction. This shows that $x^{*}$ is $c$-maximal in $P$.

\subsection{Length of simplex path}

We now present a proof of Theorem 2, which provides a bound on the length of the simplex path generated by the iterative algorithm.

Proof of Theorem Q First, note that the iterative algorithm constructs a simplex path from $x^{0}$ to the output vector. This is the path obtained by merging all the simplex paths constructed by the scaling algorithm in step 3 at each iteration. From Lemma 3 , the iterative algorithm can indeed call the scaling algorithm with input vertex the current $x^{*}$, since $x^{*}$ is feasible for problem $(\underline{\tilde{P}})$.

Next, we upper bound the length of the generated simplex path. First, note that the iterative algorithm performs at most $n$ iterations. This is because, by Lemma 6 , at each iteration the rank of the row submatrix of $A$ indexed by $\mathcal{E}$ increases by one. Therefore, at iteration $n+1$, the subspace $\left\{x \in \mathbb{R}^{n} \mid a_{i}^{\top} x=0\right.$ for $\left.i \in \mathcal{E}\right\}$ in step 1 is the origin. Hence the projection $\bar{c}$ of $c$ onto this subspace is the origin, and the algorithm terminates by returning the current vector $x^{*}$.

Each time the iterative algorithm performs step 3, it calls the scaling algorithm with input $F, x^{*}$, and $\tilde{c}$. Since $F$ is a $[0, k]$-polytope, by Proposition 1, each time the scaling algorithm is called, it generates a simplex path of length at most $n k\left(\left\lceil\log \|\tilde{c}\|_{\infty}\right\rceil+1\right)$, where $\|\tilde{c}\|_{\infty}=n^{3} k \alpha$. Since $\log \|\tilde{c}\|_{\infty} \in O(\log (n k \alpha))$, each time we run the scaling algorithm, we generate a simplex path of length in $O(n k \log (n k \alpha))$. Therefore, the simplex path generated throughout the entire algorithm has length in $O\left(n^{2} k \log (n k \alpha)\right)$.

We immediately obtain the following corollary of Theorem 2

Corollary 1. If $\alpha$ is polynomially bounded in $n, k$, then the length of the simplex path generated by the iterative algorithm is in $O\left(n^{2} k \log (n k)\right)$. If we also assume $k=1$, the length reduces to $O\left(n^{2} \log n\right)$. 


\subsection{Complexity}

In this last section, we bound the number of operations performed to construct the next vertex in the simplex path.

Remark 5. The number of operations performed by the iterative algorithm to construct the next vertex in the simplex path is polynomially bounded in $\operatorname{size}(A)$ and $\log k$. If $P$ is simple, the number of operations is in $O\left(n^{4}+n^{3} m \log ^{2}(n k \alpha)\right)$.

Proof. First, we discuss the number of operations performed in a single iteration of the iterative algorithm:

(a) In step 1, computing the projection $\bar{c}$ of $c$ onto the subspace $\left\{x \in \mathbb{R}^{n} \mid a_{i}^{\top} x=\right.$ 0 for $i \in \mathcal{E}\}$ can be done in $O\left(n^{3}\right)$ operations via Gaussian elimination.

(b) In step 2, computing the approximation $\tilde{c}$ of $\bar{c}$ can be done by binary search, and the number of comparisons required is at most $n \log \|\tilde{c}\|_{\infty}$.

(c) In step 3 we call the scaling algorithm to compute the vector $\tilde{x}$. From Remark 3 , the number of operations performed to construct the next vertex in the simplex path is polynomially bounded in $\operatorname{size}(A)$ and $\log \|\tilde{c}\|_{\infty}$. If $P$ is simple, the number of operations is $O\left(n^{2} m \log ^{2}\|\tilde{c}\|_{\infty}\right)$.

(d) At the end of step 3 we compute the vector $\tilde{y}$. From Lemma 4, the number of operations performed to compute this vector is polynomially bounded in $\operatorname{size}(A)$, and by $O\left(n m+n^{3}\right)$ if $P$ is simple.

Recall from the proof of Theorem 2 that the iterative algorithm performs at most $n$ iterations. Moreover, each vector $\tilde{c}$ computed at step 2 is such that $\log \|\tilde{c}\|_{\infty} \in O(\log (n k \alpha))$.

Let $x^{i}$ be the current vertex of the simplex path computed by the iterative algorithm, and denote by $x^{i+1}$ the next vertex in the simplex path that we will construct. In the worst case, the vertex $x^{i+1}$ is computed by the scaling algorithm in the very last iteration. Therefore, the number of operations is bounded by the product of $n$ with the sum of the operations bounds in (a) (d) above. In the general case, this number is polynomially bounded in $\operatorname{size}(A)$ and $\log \|\tilde{c}\|_{\infty}$. If $P$ is simple, this number is bounded by

$$
O\left(n \cdot\left(n^{3}+n \log \|\tilde{c}\|_{\infty}+n^{2} m \log ^{2}\|\tilde{c}\|_{\infty}+n m+n^{3}\right)\right) \in O\left(n^{4}+n^{3} m \log ^{2}\|\tilde{c}\|_{\infty}\right) .
$$

The statement follows $\operatorname{since} \operatorname{size}(A)$ is polynomial in $n, m, \log \alpha$, and $\log \|\tilde{c}\|_{\infty} \in O(\log (n k \alpha))$.

The following remark shows that if the polytope $P$ is 'well-described' by the system $A x \leq b$, then the number of operations performed by both the preprocessing \& scaling algorithm and by the iterative algorithm to construct the next vertex in the simplex path is polynomially bounded in $n, m, \log k$. In particular, it is independent on $\alpha$.

Remark 6. Assume that $P$ is full-dimensional, that each inequality in $A x \leq b$ is facetdefining, and that the greatest common divisor of the entries in each row of $A$ is one. Then, the number of operations performed by the preprocessing \& scaling algorithm and by the iterative algorithm to construct the next vertex in the simplex path is polynomially bounded in $n, m, \log k$. 
Proof. From Remark 4 and Remark 5, the number of operations performed by either algorithm to construct the next vertex in the simplex path is polynomially bounded in $\operatorname{size}(A)$ and $\log k$. Recall that $\operatorname{size}(A)$ is polynomial in $n, m$, and $\log \alpha$. Therefore, it suffices to show that $\log \alpha$ is polynomially bounded in $n$ and $\log k$.

Denote by $\varphi$ the facet complexity of $P$ and by $\nu$ the vertex complexity of $P$. From Theorem 10.2 in 23 , we know that $\varphi$ and $\nu$ are polynomially related, and in particular $\varphi \leq 4 n^{2} \nu$. Since $P$ is a $[0, k]$-polytope, we have $\nu \leq n \log k$. Due to the assumptions in the statement of the remark, and Remark 1.1 in [7], we obtain that $\log \alpha \leq n \varphi$. Hence, $\log \alpha \leq n \varphi \leq 4 n^{3} \nu \leq 4 n^{4} \log k$.

Note that the assumptions in Remark $[6$ are without loss of generality, and it is wellknown that we can reduce ourselves to this setting in polynomial time. Remark 6 then implies that, if we assume that $k$ is polynomially bounded by $n$ and $m$, then both algorithms run in strongly polynomial time.

To conclude, we highlight that all the obtained bounds on the number of operations performed by our algorithms (see Remarks 106) also depend on the number $m$ of inequalities in the system $A x \leq b$. This is in contrast with the lengths of the simplex paths, which only depend on $n$ and $k$. This difference is to be expected, since in order to determine the next vertex, the algorithm needs to read all the inequalities defining the polytope, thus the number of operations must depend also on $m$.

\section{References}

[1] D. Acketa and J. Zunić. On the maximal number of edges of convex digital polygons included into an $m \times m$-grid. Journal of Combinatorial Theory, Series A, 69(2):358 - 368, 1995.

[2] R.K. Ahuja, T.L. Magnanti, and J.B. Orlin. Network Flows: Theory, Algorithms, and Applications. Prentice Hall, Englewood Cliffs NJ, 1993.

[3] N. Alon and V.H. Vu. Anti-hadamard matrices, coin weighing, threshold gates, and indecomposable hypergraphs. Journal of Combinatorial Theory, Series A, 79:133160, 1997.

[4] A. Balog and I. Bárány. On the convex hull of the integer points in a disc. In Proceedings of the seventh annual symposium on Computational geometry, SCG '91, pages 162-165, New York, NY, USA, 1991. ACM.

[5] D. Bertsimas and J. Tsitsiklis. Introduction to Linear Optimization. Athena Scientific, Belmont, MA, 1997.

[6] M. Blanchard, J. De Loera, and Q. Louveaux. On the length of monotone paths in polyhedra. arXiv:2001.09575, 2020.

[7] M. Conforti, G. Cornuéjols, and G. Zambelli. Integer Programming. Springer, 2014. 
[8] A. Del Pia and C. Michini. On the diameter of lattice polytopes. Discrete \& Computational Geometry, 55(3):681-687, 2016.

[9] A. Deza, G. Manoussakis, and S. Onn. Primitive zonotopes. Discrete \& Computational Geometry, 60(1):27-39, July 2018.

[10] A. Deza and L. Pournin. Improved bounds on the diameter of lattice polytopes. Acta Mathematica Hungarica, 154(2):457-469, 2018.

[11] A. Deza, L. Pournin, and N. Sukegawa. The diameter of lattice zonotopes. Proceedings of the American Mathematical Society (to appear), 2020.

[12] E.A. Dinic. The method of scaling and transportation problems. Issled. Diskret. Mat. Science, Moscow. (In Russian.), 1973.

[13] J. Edmonds and R.M. Karp. Theoretical improvements in algorithmic efficiency for network flow problems. Journal of the Association for Computing Machinery, 19:248$264,1972$.

[14] A. Frank and É Tardos. An application of simultaneous diophantine approximation in combinatorial optimization. Combinatorica, 7:49-65, 1987.

[15] H.N. Gabow. Scaling algorithms for network problems. Journal of Computer and SystemSciences, 31:148-168, 1985.

[16] T. Kitahara, T. Matsui, and S. Mizuno. On the number of solutions generated by Dantzig's simplex method for LP with bounded variables. Pacific Journal of Optimization, 8(3):447-455, 72012.

[17] T. Kitahara and S. Mizuno. A bound for the number of different basic solutions generated by the simplex method. Mathematical Programming, Series A, 137:579586, 2013.

[18] P. Kleinschmidt and S. Onn. On the diameter of convex polytopes. Discrete Mathematics, 102:75-77, 1992.

[19] A.K. Lenstra, H.W. Lenstra, and L. Lovász. Factoring polynomials with rational coefficients. Mathematische Annalen, 261:515-534, 1982.

[20] S. Mizuno. The simplex method using Tardos' basic algorithm is strongly polynomial for totally unimodular LP under nondegeneracy assumption. Optimization Methods and Software, 31(6):1298-1304, 2016.

[21] S. Mizuno, N. Sukegawa, and A. Deza. An enhanced primal-simplex based Tardos' algorithm for linear optimization. Journal of the Operations Research Society of Japan, 61(2):186-196, 2018.

[22] D.J. Naddef. The Hirsch conjecture is true for $(0,1)$-polytopes. Mathematical Programming, 45:109-110, 1989. 
[23] A. Schrijver. Theory of Linear and Integer Programming. Wiley, Chichester, 1986.

[24] A. Schrijver. Combinatorial Optimization. Polyhedra and Efficiency. Springer-Verlag, Berlin, 2003.

[25] A.S. Schulz, R. Weismantel, and G.M. Ziegler. 0/1-integer programming: Optimization and augmentation are equivalent. In Proceedings of ESA '95, pages 473-483, 1995.

[26] E. Tardos. A strongly polynomial algorithm to solve combinatorial linear programs. Operations Research, 34(2):250-256, 1986.

[27] T. Thiele. Extremalprobleme für Punktmengen. Master's thesis, Freie Universität Berlin, 1991.

[28] Günter M. Ziegler. Lectures on 0/1-polytopes. In Gil Kalai and Günter M. Ziegler, editors, Polytopes - Combinatorics and Computation, pages 1-41. Birkhäuser Basel, Basel, 2000. 